\section{Regarding Pharmacology, Part 3A}

TO THE EDITOR: I read "Pharmacology, Part 3A" in the December 2018 issue of the Journal of Nuclear Medicine Technology by Geoffrey M. Currie (1). I am concerned about one aspect of the discussion, specifically that of cholecystokinin, or its analog, sincalide (Kinevac; Bracco Diagnostics, Inc.), and its proper clinical use. The article states that the recommended dose is $0.02 \mu \mathrm{g}$ and should be infused over $3-5 \mathrm{~min}$. This method of infusion has been discredited, dating back to 1992 (2). Three-min infusions have been shown to have a very wide range in gallbladder ejection fraction response in healthy subjects and because of this, normal values could not be determined 2001 (3). In addition, $50 \%$ of these healthy subjects develop nausea and abdominal cramping. When the same healthy subjects received slower rates of infusions of 30-60 min, there was much less variability in response and normal values could be determined, although they were different for the 2 different infusion rates. Furthermore, no subjects had adverse symptoms.

These findings led to a multicenter investigation sponsored by the Gastrointestinal Council of the Society of Nuclear Medicine (now the General Nuclear Medicine Council of the Society of Nuclear Medicine and Molecular Imaging [SNMMI]) to determine the optimal method of cholecystokinin-cholescintigraphy infusion by comparing 15-, 30-, and 60-min infusions of sincalide in 60 healthy subjects at 4 institutions (4). The 15-min infusion showed the widest range of response, and similarly clinically useful normal values could not be determined. Some of these healthy subjects had nausea and or abdominal cramping. The 30-min infusion showed less variability; however, normal values could still not be calculated. The 60 -min infusion showed the least variability in these healthy subjects, and normal values could be determined ( $\geq 38 \%$ gallbladder ejection fraction). No patients had adverse symptoms with the 30 - or 60 -min infusion methods.

These published data strongly suggest that patients given short infusions may have false-positive studies (low gallbladder ejection fractions but without disease) and end up erroneously going to surgery to have their gallbladder removed. The data also suggest that the adverse symptoms are caused by the method of infusion and unrelated to the presence or absence of disease.

The SNMMI Practice Guideline for Hepatobiliary Scintigraphy 4.0, published in 2010, recommends a slow infusion of sincalide (5). Subsequently an interdisciplinary panel consisting of 12 gastroenterologists, surgeons, and nuclear medicine physicians met and then published recommendations in 2011 to standardize sincalide infusion methodology at all centers and specifically recommended the slow infusion method of $0.02 \mu \mathrm{g}$ over $60 \mathrm{~min}(6)$.

\section{REFERENCES}

1. Currie GM. Pharmacology, Part 3A: interventional medications in renal and biliary imaging. J Nucl Med Technol. 2018;46:326-334.

COPYRIGHT (C) 2019 by the Society of Nuclear Medicine and Molecular Imaging.
2. Ziessman HA, Fahey FH, Hixson DJ. Calculation of a gallbladder ejection fraction: advantage of continuous sincalide infusion over the three-minute infusion method. J Nucl Med. 1992;33:537-541.

3. Ziessman HA, Muenz LR, Agarwal AK, Zaza AAM. Normal values for sincalide cholescintigraphy: comparison of two methods. Radiology. 2001;221:404-410.

4. Ziessman HA, Tulchinsky M, Lavely WC, et al. Sincalide-stimulated cholescintigraphy: a multicenter investigation to determine optimal infusion methodology and gallbladder ejection fraction normal values. J Nucl Med. 2010;51: 277-281.

5. Tulchinsky M, Ciak BW, Delbeke D, et al. SNM practice guideline for hepatobiliary scintigraphy 4.0. J Nucl Med Technol. 2010;38:210-218.

6. DiBaise JK, Richmond BK, Ziessman HA, et al. Cholecystokinin-cholescintigraphy in adults: consensus recommendations of an interdisciplinary panel. Clin Gastroenterol Hepatol. 2011;9:376-384.

Harvey A. Ziessman

Johns Hopkins Medical Institute 601 North Caroline St., Suite 3231

Baltimore, MD 21278

E-mail: hziessm1@jhmi.edu

Published online Apr. 24, 2019.

DOI: 10.2967/jnmt.119.225706

\section{Reply: Regarding Pharmacology, Part 3A}

REPLY: Thank you, Harvey, for drawing this to our attention, The discussion around sincalide (Kinevac; Bracco) dose rates is not lost on me, and I wrestled with this for some time with the original paper (1). I have provided a correction (2) to the section, recognizing the widespread use of the traditional 3- to 5-min infusion method and highlighting the more physiologic response the longer 60-min infusion provides.

I recognize the great work your team has done in validating longer infusion times and the recommendations of the consensus committee. Nonetheless, the Journal of Nuclear Medicine Technology has an international readership and some consideration needs to be given to global practices. Although I agree that the 60 -min infusion method is a more physiologic response, I have some reservations about some of the other conclusions drawn in the literature and as such, aimed to uncomplicate that aspect of the paper (1).

The bulk of the literature, including yours, is built on the premise that there is variability in the normal value among healthy subjects $(35 \%)$ and as a result, potential for false-positives. The issue I see with the gallbladder ejection fraction (GBEF) quantitation is the interpretation. For me, $35 \%$ is the value above which we can be reasonably certain (95\%) represents absence of gallbladder dysfunction (even if other biliary disease is present). It does not and should not suggest that a value under $35 \%$ equates to abnormality or the notion of false-positives. Similar to the multiple ranges for post-Lasix renal imaging ( $<10 \mathrm{~min}$ is normal, $>20 \mathrm{~min}$ is obstructed, and $10-20 \mathrm{~min}$ is dilated but not obstructed), biliary imaging needs a lower range, a second value representing the boundary below which we are $95 \%$ certain gallbladder dysfunction is present. The literature discrediting the 3- to 